\title{
CONGRUENCE AND ONE-DIMENSIONALITY OF METRIC SPACES
}

\author{
LUDVIK JANOS
}

(Communicated by Dennis Burke)

\begin{abstract}
Two subsets $A$ and $B$ of a metric space $(X, d)$ are said to be congruent if there is a bijection between them which preserves the distance $d$. We show that if a separable locally compact metric space is such that no distinct subsets of cardinality 3 are congruent then its dimension is $\leq 1$. We also show that the real line $\mathbf{R}$ can be given a compatible metric with this property.
\end{abstract}

1. Introduction. We say that two subsets $A, B$ of a metric space $(X, d)$ are congruent if there exists a bijection between them which preserves the distance $d$. Using the notion of congruence our previous result [2] says that a nonempty separable metrizable space is zero-dimensional if and only if it admits a metric relative to which no two distinct sets of cardinality 2 are congruent. Our objective in this note is to explore the case where the cardinality of the sets mentioned above is 3 . We prove

THEOREM 1. If $X$ is a locally compact separable metric space having a metric $d$ such that no two distinct subsets of $X$ of cardinality 3 are congruent relative to $d$, then $\operatorname{dim}(X) \leq 1$.

THEOREM 2. The real line $\mathbb{R}$ can be given a compatible metric $d$ so that $(\mathbb{R}, d)$ contains no two distinct congruent subsets of cardinality 3.

2. Proof of the theorems. If $x_{1}, x_{2} \in X$ are two distinct points of a metric space $(X, d)$ we denote by $B\left(x_{1}, x_{2}\right)$ the bisector set defined as $\{y: y \in X$ and $\left.d\left(x_{1}, y\right)=d\left(x_{2}, y\right)\right\}($ cf. $[\mathbf{3}, \mathbf{4}$, or $\mathbf{5}])$.

LEMMA 2.1. Let $X$ be a separable metrizable space. Then, $\operatorname{dim}(X) \leq n$ if and only if $X$ has an admissible totally bounded metric $d$ such that if $B$ is a d-bisector set in $X$ then $\operatorname{dim}(B) \leq n-1$.

ProOF. This is Theorem 1 of [4].

As a corollary to this lemma we prove

LEMMA 2.2. Let $(X, d)$ be a totally bounded metric space containing no two distinct congruent sets of cardinality 3 . Then $\operatorname{dim}(X) \leq 1$.

ProOF. If $X$ is empty or if it contains only one point then $\operatorname{dim}(X) \leq 0$. So assume $X$ contains at least two points and let $B=B\left(x_{1}, x_{2}\right)$ be a bisector set of

Received by the editors May 7, 1987.

1980 Mathematics Subject Classification (1985 Revision). Primary 54F45, 54F50; Secondary 54E35, 54E40. 
$X$. We observe that the set $B$ cannot contain more than one point since if $y_{1}$, $y_{2} \in B$ were two distinct points then $\left\{x_{1}, y_{1}, y_{2}\right\}$ and $\left\{x_{2}, y_{1}, y_{2}\right\}$ would be two distinct congruent sets of cardinality 3 which is contrary to our hypothesis. Thus we have that $\operatorname{dim}(B) \leq 0$ for every bisector set of $X$ and Lemma 2.1 implies that $\operatorname{dim}(X) \leq 1$ which was to be proved.

The proof of our Theorem 1 now follows as a corollary to Lemma 2.2. Since $(X, d)$ is a locally compact separable metric space each point $x \in X$ is contained in a precompact open subset $U \subseteq X$ so that the restriction of $d$ to $U$ is totally bounded. Since the dimension is a local property we conclude that $\operatorname{dim}(X) \leq 1$.

To prove our Theorem 2 we need some notation. We denote by $\mathbb{R}^{2}$ the Euclidean plane with the usual Euclidean metric on it. Let $I$ denote the group of all orientation preserving isometries of $\mathbb{R}^{2}$, i.e., the proper motions and let $I^{*}$ be the group of all isometries of $\mathbb{R}^{2}$ including reflexions. Let $P$ denote the graph $\left\{\left(x, x^{2}\right): x \in \mathbb{R}\right\}$ of the parabola $y=x^{2}$ in $\mathbb{R}^{2}$ and let $P^{+} \subseteq \mathbb{R}^{2}$ denote the following subset of it: $\left\{\left(x, x^{2}\right): x>0\right\}$. We observe that $P^{+}$is homeomorphic to $\mathbb{R}$ and we shall prove that it has the desired property relative to the Euclidean metric of $\mathbb{R}^{2}$.

LEMMA 2.3. Let $\left\{A_{1}, A_{2}, A_{3}\right\}$ and $\left\{B_{1}, B_{2}, B_{3}\right\}$ be two distinct subsets of $P^{+}$ of cardinality 3 . Then these sets cannot be congruent.

ProOF. We give an indirect proof, assuming they are congruent. Then, as we know (cf. [1]) the isometry between $\left\{A_{1}, A_{2}, A_{3}\right\}$ and $\left\{B_{1}, B_{2}, B_{3}\right\}$ extends uniquely to an element $T \in I^{*}$. Now we distinguish two cases: Case (1) where $T$ is a proper motion. Let the points $A_{i}=\left(x_{i}, x_{i}^{2}\right)$ and $B_{j}\left(y_{j}, y_{j}^{2}\right)$ be indexed so that for their first coordinates $x_{i}$ and $y_{j}$ we have $0<x_{1}<x_{2}<x_{3}$ and $0<y_{1}<y_{2}<y_{3}$ respectively. Then, since $T$ is order-preserving we have: $B_{i}=T A_{i}$ for $i=1,2,3$. Without loss of generality we may assume $x_{1} \leq y_{1}$ from which one easily deduces that $x_{2} \leq y_{2}$ and $x_{3} \leq y_{3}$. An elementary computation shows that the negatively taken tangent of the angle at the vertex $A_{2}$ of the triangle $\left\{A_{1}, A_{2}, A_{3}\right\}$ equals

$$
a_{13}\left[1+\left(x_{1}+x_{2}\right)\left(x_{2}+x_{3}\right)\left(1+x_{1}^{2}+2 x_{1} x_{3}+x_{3}^{2}\right)\right]^{-1}
$$

where $a_{13}=\left[\left(x_{3}-x_{1}\right)^{2}+\left(x_{3}^{2}-x_{1}^{2}\right)^{2}\right]^{1 / 2}$ is the distance between $A_{1}$ and $A_{3}$. Since both of these quantities are preserved under $T$ we obtain the equality

$$
\begin{aligned}
& 1+\left(x_{1}+x_{2}\right)\left(x_{2}+x_{3}\right)\left(1+x_{1}^{2}+2 x_{1} x_{3}+x_{3}^{2}\right) \\
& \quad=1+\left(y_{1}+y_{2}\right)\left(y_{2}+y_{3}\right)\left(1+y_{1}^{2}+2 y_{1} y_{3}+y_{3}^{2}\right) .
\end{aligned}
$$

But since the expression on the left is an increasing function of $x_{1}, x_{2}$ and $x_{3}$ and since $y_{1} \geq x_{1}, y_{2} \geq x_{2}, y_{3} \geq x_{3}$ the equality would imply $x_{i}=y_{i}$ for $i=1,2,3$, which is impossible since the sets $\left\{A_{1}, A_{2}, A_{3}\right\}$ and $\left\{B_{1}, B_{2}, B_{3}\right\}$ are distinct. So it remains to investigate Case (2), where $T$ is a reflexion. Let $L$ be the line in $\mathbb{R}^{2}$ which is pointwise invariant under $T$. We observe that the set $\left\{A_{1}, A_{2}, A_{3}, B_{1}, B_{2}, B_{3}\right\}$ has cardinality at least 4 and that it is contained in the intersection $P^{+} \cap T P^{+}$. We also observe that the line $L$ would intersect the parabola $P$ in two points $C_{1}, C_{2}$ where $C_{1} \in P^{+}$and $C_{2} \notin P^{+}$. Thus, the set $\left\{A_{1}, A_{2}, A_{3}, B_{1}, B_{2}, B_{3}, C_{2}\right\}$ with cardinality at least 5 would be in the intersection $P \cap T P$ which contradicts the fact that two quadratic curves have at most 4 common points. So we arrived at the desired contradiction in both cases which concludes the proof of our lemma.

Identifying the line $\mathbb{R}$ with the set $P^{+}$and taking for $d$ the restriction of the Euclidean metric of $\mathbb{R}^{2}$ to $P^{+}$we obtain the proof of our Theorem 2 . 
REMARK. The fact that $\mathbb{R}$ can be remetrized in such a manner that no two distinct sets of cardinality 3 are congruent supports our belief that also the converse of Theorem 1 is true, namely that if a separable metrizable space has dimension $\leq 1$ then it has a metric with this property. One way to show it would be to prove that the universal 1-dimensional subset of $\mathbb{R}^{3}$, the Sierpiński cube, has such a metric. In [3] is shown that there exists a subset of $\mathbb{R}$ containing no two distinct congruent subsets of cardinality 2 which is homeomorphic to the Cantor set $C$. We conjecture that there exists a set $S^{*} \subseteq \mathbb{R}^{3}$ homeomorphic to the Sierpinski set $S$ such that for every nonidentical isometry $T$ of $\mathbb{R}^{3}$ the cardinality of the intersection $S^{*} \cap T S^{*}$ is at most 2 if $T$ is a proper motion and at most 3 if it is a reflexion. This would imply that the set $S^{*}$ contains no two distinct congruent subsets of cardinality 3 .

\section{REFERENCES}

1. L. M. Blumenthal, Theory and applications of distance geometry, Oxford Univ. Press, 1953.

2. L. Janos, A metric characterization of zero-dimensional spaces, Proc. Amer. Math. Soc. 31 (1972), 268-270.

3. __ Rigid subsets in Euclidean and Hilbert spaces, J. Austral. Math. Soc. 20(1975), 66-72.

4. L. Janos and H. Martin, Metric characterizations of dimension for separable metric spaces, Proc. Amer. Math. Soc. 70 (1978), 209-212.

5. J. Nagata, Modern dimension theory, rev. ed., Heldermann-Verlag, Berlin.

Department of Mathematics and Computer SCience, California State UniVERSITY, LONG BEACH, CALIFORNIA 90840 